CT Angiogram showed upper periaortic soft tissue surrounding the origin of the coeliac axis and superior mesenteric artery (SMA) causing marked stenosis of the SMA.

A working diagnosis of an inflammatory or infective Aortitis involving the proximal intra-abdominal Aorta, extending from the diaphragm to the renal vessels was made.

An extensive infectious work up was performed which proved negative including exclusion of tuberculosis.

C3 and IgA were marginally raised. Serum Anti Nuclear Antibodies, Anti-Double Stranded DNA Antibodies, Rheumatoid factor and urinary catecholamines were all within normal limits.

PET scan confirmed uptake in the proximal abdominal aorta with associated periaortic soft tissue suggestive of Aortitis.

A diagnosis of Takayasu arteritis- large vessel granulomatous vasculitis, was made.

Treatment was instigated with high dose intravenous methylprednisolone for three days followed by high dose oral prednisolone and subcutaneous methotrexate at a dose of $15 \mathrm{mg} / \mathrm{m}^{2}$ weekly.

Inflammatory markers slowly began to normalise with immunosuppressive treatment. Follow up ultrasound at one month showed interval improvement in the aortic mass with increase in the aortic lumen size. On corticosteroid wean a further ultrasound 6 weeks later showed no improvement in the mass and was associated with a rise in inflammatory markers. Biologic therapy with adalimumab subcutaneously has since been added with plan for serial imaging to assess response to therapy.

Conclusion This is a rare presentation of a large vessel vasculitis- Takayasu arteritis in a male child of Caucasian origin.

\section{P70 TWIST AND SHOUT: DELAYED PRESENTATION OF TESTICULAR TORSION IN A 14 YEAR OLD}

\footnotetext{
1,2Gerard McGuinness*, ${ }^{2}$ Adrian Fuentes, ${ }^{2}$ William Shields. ${ }^{1}$ Royal College of Surgeons in Ireland, St. Stephen's Green, Dublin, Ireland; ${ }^{2}$ Department of Urology, University Hospital Waterford, Waterford, Ireland
}

\subsection{6/archdischild-2019-epa.425}

Introduction Testicular torsion is an acute surgical emergency, with an approximate 6 hour window from onset of symptoms, to irreversible damage to the testicle. ${ }^{1}$ Trauma associated testicular torsion is a well recognised complication of blunt scrotal trauma, accounting for $4-8 \%$ of all torsions. ${ }^{2,3}$ Delays in recognition of the condition, and subsequent delayed surgical exploration, may necessitate orchidectomy, and risks impairment of future fertility. ${ }^{4}$ Patient factors associated with delay in presentation include embarrassment around pain in the genitals, and lack of awareness of the condition. ${ }^{5}$

Case description A 14 year old Irish boy, with a previous history of bilateral retractile testes, presented to the Emergency department with a three day history of severe left sided scrotal pain and swelling following a traumatic injury to the perineum. The patient was cycling off-road three days prior to presentation, and suffered a cross-bar related injury to the scrotum and perineum. He felt immediate left testicular pain, however believed this to be related to bruising of his testicle. The pain progressively worsened over the following three days, and presented to hospital once the pain had become unbearable.
Examination demonstrated a tender, erythematous, and swollen left hemiscrotom with an absent cremasteric reflex on the left side. The right testicle was retractile, but present within the right hemiscrotom, with a positive cremasteric reflex. US of the scrotom demonstrated an enlarged, oedematous left testicle, with absent internal vasculature, and a normal appearing right testicle. At operation, the left testicle was found to be necrotic and non-viable, and so we proceeded with a left orchidectomy. After discussion with his parents, we decided to proceed with a right sided orchidopexy with threepoint fixation of his remaining testicle. The patient was discharged home later that day on a course of oral antibiotics.

Discussion Time is of utmost importance in cases of testicular torsion, with early presentation, and early surgical exploration the most important factors in testicular salvage. ${ }^{3}$ It is easy to attribute post-traumatic testicular pain and swelling to the initial insult, however, a high index of suspicion is required to out-rule trauma associated testicular torsion. Early education around testicular health, an understanding that scrotal pain requires swift medical evaluation, and that delays in evaluation may impair future fertility, are important messages to convey to paediatric populations, and should be included in sexual health education for males.

\section{P71 SKELETAL DYSPLASIA- CASE REPORT OF AN INFANT WITH THANATOPHORIC DYSPLASIA}

Siobhan McCormack*, Claire Thompson, Kerrie Hennigan, Rizwan Khan, Niazy Al-assaf. Department of Neonatology, University Maternity Hospital Limerick, Limerick, Ireland

\subsection{6/archdischild-2019-epa.426}

Background An infant with respiratory failure and features of skeletal dysplasia

Case report A male infant dichorionic twin weighing $2.3 \mathrm{Kg}$ with antenatally suspected skeletal dysplasia had initial examination findings of frontal bossing, a flat nasal bridge, a wide anterior fontanelle and shortened upper and lower limbs along with a markedly narrow chest and short ribs. His systemic exam revealed no other abdominal or cardiovascular abnormalities. His twin had normal growth parameters and phenotypic appearance.

He was intubated within a few minutes of life and commenced on high frequency oscillatory ventilation. A skeletal survey confirmed his thorax to be narrow with shortened ribs and handlebar clavicles. He also had vertebral abnormalities with platyspondyly and U-shaped vertebra. His limbs showed generalised micromelia with short bowed femurs and humerii. He had no pneumothoraces.

The infant displayed progressive respiratory failure despite maximum oxygen concentration at 50 hours of life and interventions were discontinued due to futility. His clinical and radiological findings were most in keeping with Thanatophoric dysplasia I and this was confirmed genetically on microarray showing heterozygous FGFR3 mutation.

Discussion Thanatophoric dysplasia is the most frequent form of lethal osteochondrodysplasia with an estimated incidence of 1 in 60,000 births. ${ }^{1}$ There are two subtypes: Type ${ }^{1}$ is characterised by a short, curved femur, and a straighter femur with cloverleaf skull characterises type II. $^{2}$ Affected infants show marked underdevelopment of the skeleton and short limbs due to sporadic mutations of fibroblast growth factor receptor 3 gene (FGFR3). ${ }^{3}$ Infants are usually stillborn or die shortly 